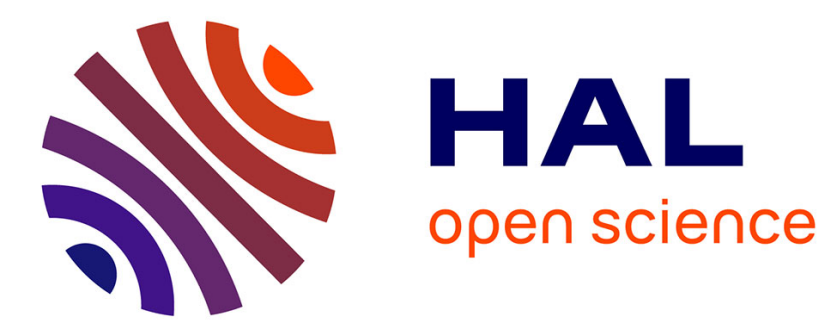

\title{
A Program to Analyse the Origin of Noise in Ultra- Stable Quartz Crystal Resonators
}

\author{
Serge Galliou, Fabrice Sthal, Xavier Vacheret, Rémi Brendel, Patrice
}

Salzenstein, Enrico Rubiola, Gilles Cibiel

\section{- To cite this version:}

Serge Galliou, Fabrice Sthal, Xavier Vacheret, Rémi Brendel, Patrice Salzenstein, et al.. A Program to Analyse the Origin of Noise in Ultra- Stable Quartz Crystal Resonators. IEEE Frequency Control Symposium \& European Time and Frequency Forum, Jun 2007, Geneva, Switzerland. pp.1176-1181, 10.1109/FREQ.2007.4319263 . hal-00154309

\section{HAL Id: hal-00154309 \\ https://hal.science/hal-00154309}

Submitted on 13 Jun 2007

HAL is a multi-disciplinary open access archive for the deposit and dissemination of scientific research documents, whether they are published or not. The documents may come from teaching and research institutions in France or abroad, or from public or private research centers.
L'archive ouverte pluridisciplinaire HAL, est destinée au dépôt et à la diffusion de documents scientifiques de niveau recherche, publiés ou non, émanant des établissements d'enseignement et de recherche français ou étrangers, des laboratoires publics ou privés. 


\section{A Program to Analyse the Origin of Noise in Ultra- Stable Quartz Crystal Resonators}

\author{
S. Galliou, F. Sthal, X. Vacheret, R. Brendel, \\ P. Salzenstein, E. Rubiola \\ Time and Frequency Dept. \\ FEMTO-ST Institute, UMR CNRS 6174 \\ Besançon, France \\ serge.galliou@femto-st.fr
}

\author{
G. Cibiel \\ Microwave and Time-Frequency Dept. \\ CNES \\ Toulouse, France \\ gilles.cibiel@cnes.fr
}

\begin{abstract}
In the mid 90s the quartz crystal oscillator attained a stability in the upper $10^{-14}$ (flicker floor of the Allan deviation $\sigma_{y}(\tau)$, which occurs at $\left.\tau=1 . .10 \mathrm{~s}\right)$. As a matter of fact, the highest stability was obtained with bulk-acoustic-wave quartz crystal resonators at $5 \mathrm{MHz}$ and at $10 \mathrm{MHz}$. Since, the research for higher stability seems to be at a standstill, while space applications are more and more demanding.

FEMTO-ST Institute have started a research program on the origin of noise in $5 \mathrm{MHz}$ and $10 \mathrm{MHz}$ quartz crystal resonators, managed by The Centre National d'Etudes Spatiales (CNES). Several European manufacturers of high-stability resonators and oscillators participate. This article reports on the present status and on the future plans of this program.

The first part consists in the analysis of the sensitivity of selected resonators to various externally-controlled parameters, like temperature, drive power, load impedance, series capacitance.

The second part, planned, consists of listing the possible causes of noise, and of modeling their effects on frequency stability.

Tests and measurements are mainly performed on an advanced phase noise measurement system, recently set up for this program.

Of course, this program is a unique opportunity to test various batches of $5 \mathrm{MHz}$ and $10 \mathrm{MHz}$ resonators provided by the industrial partners.
\end{abstract}

\section{INTRODUCTION}

To achieve the selected missions, space applications of precise localization, navigation and sciences require frequency stabilities and spectral purity that dramatically increase. It is the case of clocks on board for PHARAO missions, and ultra stable oscillators (USO) for DORIS programs, for which the required frequency stability is about or better $1.10^{-13}$ for $\tau$ from 1 to $10 \mathrm{~s}$. These stabilities are required either for ultra stable quartz crystal oscillators (DORIS program) or atomic clocks (PHARAO project) in which the ultra stable oscillator (USO) gives the limitation of stability. In these cases, very low noise USO are required, and for this reason it is necessary to select and control the sources of noise of the quartz crystal resonator.

DORIS program (Doppler Orbitography and Radiopositioning Integrated in Space) is a Doppler satellite tracking system developed for precise determination and precise ground location. Its ultimate aim is to achieve an accuracy of one centimetre. This instrument is on-board of TOPEX-POSEIDON, JASON1 and ENVISAT altimetric satellites, it also flew with SPOT series. Future DORIS will be embarked on JASON2, PLEIADES, ALTIKa, HY2, CRYOSAT2.

The feasibility of quartz crystal oscillators exhibiting relative frequency stabilities that can reach $5 \cdot 10^{-14}$ over a few seconds was clearly proven [3]. Unfortunately, their reproducibility is still far from being systematic whereas the demand is more and more severe in terms of specifications. Indeed, it is clear that the precision of localization by means of satellites or the precision of clocks used for synchronization in telecommunication systems results in increasingly severe requirements on the performances of the USO, at the limit of the know-how of today.

To reach the level of performances required by current projects (PHARAO, JASON2, PLEIADES, ALTIKa, HY2, CRYOSAT2) and futures projects (mainly altimetric missions which need DORIS instrument), it is necessary to sort all the quartz crystal devices one by one, in terms of noise. The outputs are extremely weak (a few \%) to obtain performances such as those required by PHARAO $\left(7.10^{-14}\right.$ for $\left.\tau=1 \mathrm{~s}\right)$. To hope to improve the stability of USO, it is necessary to continue and go further into works of investigations on the origin of noise in resonators. Currently, the distribution of USO stability is centered on $1 \cdot 10^{-13}$ over a few seconds, for best crystal units from major European manufacturers. It is necessary to be able to shift it to 7 , even $5 \cdot 10^{-14}$, in production.

This program is managed by the French space agency CNES (Centre National d'Etudes Spatiales) of Toulouse, is led by the FEMTO-Institute (UMR CNRS 6174) and involved major European manufacturers (AR Electronics Besançon France, Oscilloquartz Neuchatel Switzerland, Rakon Argenteuil France, Temex Troyes France). 


\section{GUIDING LINES OF THE PROGRAM}

The problem is complex and requires a long-term study to end up on a noticeable improvement of the manufacturing processes of 5 and $10 \mathrm{MHz}$ ultrastable resonators of $10^{-14}$ grades. One (ambitious) aim of the study is to develop a model of the resonator noise from investigations on the physical origins of its noise. This will be performed in two main steps.

In a first step of 18 months, and before investigating physical origins of noise in resonators, we schedule to investigate correlations between actual geometrical data, external parameters of influence and the measured resonator parameters. For this study according to a macroscopic point of view, four research axes are scheduled to improve the understanding of resonator noise:

- Study of resonators sensitivity to the drive level,

- Study of the load influence,

- Study of the influence of the temperature (dynamic effect, influence of the operating point versus the turn over temperature), up).

Study of the influence of the frequency shift (pull-

These actions are based on an intensive use of the new bench for phase noise measurement recently developed at the FEMTO-ST Institute [2]. Analysis will be performed from measurements made on samples of ultrastable resonators of various industrial batches.

The second step will consist in building a noise model for bulk acoustic wave resonators from a more microscopic basis. Previous actions are necessary to any further development of such a model. Their results will condition the second step orientation. The latter will include a PhD work over duration of about 36 months.

\section{MEANS OF MEASUREMENT}

The first step of this study is essentially based on the use of a phase noise measurement bench (Fig. 1) recently developed at the FEMTO-ST institute, and still improved everyday $[1,2,4]$.

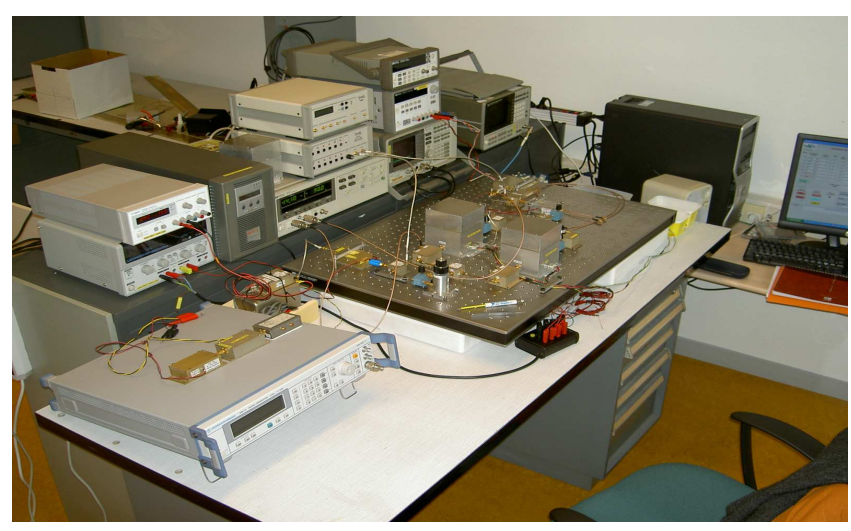

Figure 1. Measurement bench.

The general idea of this passive method (see Fig. 2) consists in reducing the noise of the source as much as possible. Indeed, when resonators exhibit a very weak noise, the noise of the source is always higher than that of the quartz crystal resonator. Thus, the direct feeding of the driving source signal through only one resonator does not permit to extract the resonator noise from the output resulting noise. On the other hand, the source signal can be subtracted when passing though two identical arms equipped with identical resonators (the devices under tests (DUT)). Then the contribution of the source is cancelled while inner noise of both resonators is preserved because one resonator noise is different from the other one. When the carrier suppression is achieved (less than $-75 \mathrm{dBc}$ is acceptable), the resulting signal only made up noise from both resonators, is strongly amplified and mixed with the source signal to be shifted down to the low frequency domain and eventually processed by the spectrum analyzer. In such a way, noise to be measured from both resonators can be brought up at a higher level than the driving source noise. Moreover, the noise floor of the bench can be measured with resistors substituted for crystal resonators.

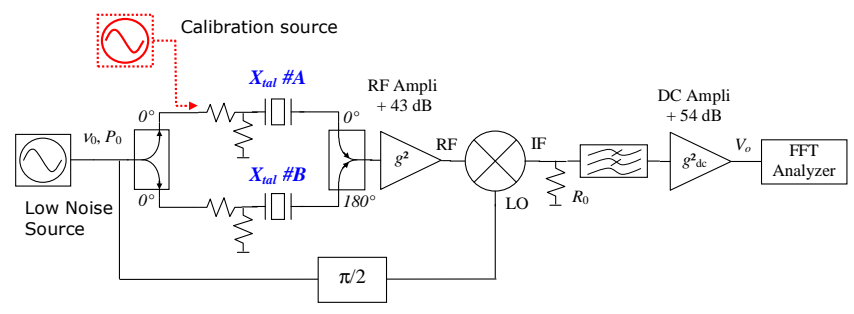

Figure 2. Principle of the measurement bench.

Figure 3 shows a typical result. The measured $\mathrm{f}^{-1}$ phase noise visible into the resonator bandwidth can be interpreted as a frequency to phase conversion. Basically, the frequency flicker noise generated by a resonator would be filtered by its own transfer function.

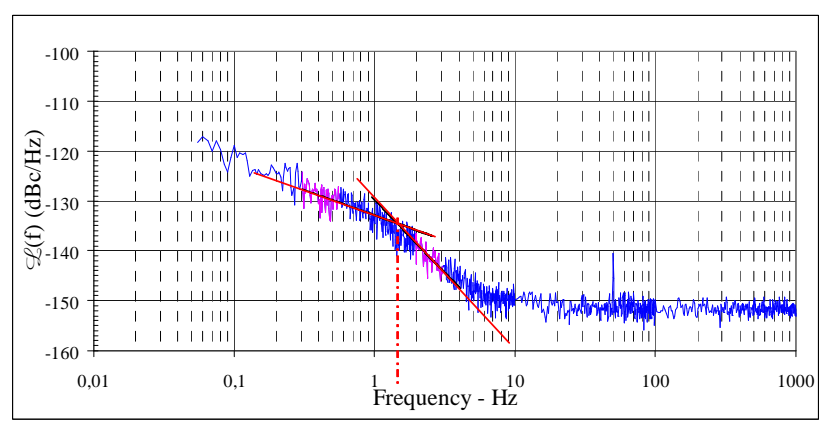

Figure 3. Typical phase noise result from two $5 \mathrm{MHz}$ resonators under test.

Two data are extracted from this graph:

$1 /$ the resonator bandwidth equal to two times the intersection frequency of the $\mathrm{f}^{-1}$ and $\mathrm{f}^{-3}$ slopes. It gives the loaded Q-factor and, thus, the conversion coefficient of the frequency noise into phase noise.

$$
S_{y}(f)=\left(\frac{1}{2 Q_{L}}\right)^{2} S_{\phi}(f)
$$


$2 /$ the measured value at $1 \mathrm{~Hz}$ from the carrier gives the level of the resulting phase noise $\left(S_{\phi}(f=1 H z)\right)$.

As a consequence, according to the following path (2), the Allan deviation of an oscillator equipped with such a noisy resonator could be calculated, assuming that the dominant phase noise in the oscillating loop is that of the resonator:

$$
\begin{aligned}
& S_{\phi}(f=1 \mathrm{~Hz}) \Rightarrow S_{y}(f=1 \mathrm{~Hz}) \\
& \Rightarrow \sigma_{y} \text { floor }=2 \ln 2 \cdot S_{y}(f=1 \mathrm{~Hz})
\end{aligned}
$$

Before the noise measurement, calibration must be performed by means of a calibrated noise or sideband modulation (at $0.4 \mathrm{~Hz}$ in our case. See Fig. 2).

Obviously, preliminary tunings are also necessary: impedance matching, series capacitor adjustment of each resonator to be at resonance at the driving source frequency, operating temperatures of each resonator. The latter have to be tuned carefully at the turning points of each frequencytemperature curve.

Presently, the resonator ovens exhibit a temperature stability of $2 \mu \mathrm{K}$ over a measuring time of $1 \mathrm{~s}$. The tuning capacitor of each resonator is also temperature-controlled. This guarantees a relative frequency stability floor better than $1 \cdot 10^{-14}$ for the usual operating conditions of the bench.

Main features of our bench as completed today are the following:

- Standard operating frequencies: $5 \mathrm{MHz}$ and $10 \mathrm{MHz}$ (at other frequencies, results depend on the driving source stability), $\mu \mathrm{W}$,

- Noise floor: -148 dBc/Hz @ $25 \mu \mathrm{W},-154 \mathrm{dBc} / \mathrm{Hz} @ 100$

- Temperature tuning of crystal ovens: by steps of $0.05^{\circ} \mathrm{C}$ over the temperature range $\left[70^{\circ} \mathrm{C}, 90^{\circ} \mathrm{C}\right]$,

- Frequency tuning $1 /$ of each arm with: a few $\mathrm{pF} \leq \mathrm{Ct} \leq \mathrm{a}$ few $10 \mathrm{pF}, 2 /$ of the source frequency at $\leq 0.01 \mathrm{~Hz}$.

- Ultimate Allan deviation $\sigma_{\mathrm{y}}(\tau)=1 \cdot 10^{-14}$ for $\tau=1 \mathrm{~s}$.

Details are given in [2]

\section{TASKS DESCRIPTION}

The work of the first stage has been divided in tasks according to their topics but, in fact, they overlap each other.

\section{A. Task 1: provisioning and fitting of high quality resonators}

Ideally, it would be interesting to analyze couples of resonators representative of various manufacturing processes and various types of resonators. This opportunity exists because several industrial partners are involved in the first part of the program. Indeed, the study will include measurements on the following samples:

- 5 MHz BVA type resonators provided by Oscilloquartz, Neuchâtel, Switzerland,

- 5 and $10 \mathrm{MHz}$ QAS type resonators from Rakon, Argenteuil, France,
- "conventional" $10 \mathrm{MHz}$ resonators from AR Electronic, Besançon, France

- $10 \mathrm{MHz}$ BVA resonators manufactured at the Institute FEMTO-ST, Besançon, France,

- "conventional" $10 \mathrm{MHz}$ resonators from TEMEX Frequency, Troyes, France.

In practice, the implementation of those resonators into the high stability bench ovens is not so easy because of their packaging variety, as shown in figure 4. Initially, ovens have been designed for homemade $10 \mathrm{MHz}$ BVA type resonators (cold weld $\mathrm{HC} 40$ enclosures of height $12 \mathrm{~mm}$ and diameter 20 $\mathrm{mm}$ ) (fig 4). Whereas HC6 packages can easily be put in with minor modifications, on the other hand bigger cases such as $\mathrm{HC} 12$ or those of $5 \mathrm{MHz}$ BVA resonators are more difficult to insert without reducing thermal performances of the ovens.

Specific ovens with measurement adaptations have been performed in this last case, to progress despite the limited length of time of 18 months for the first step of this program.

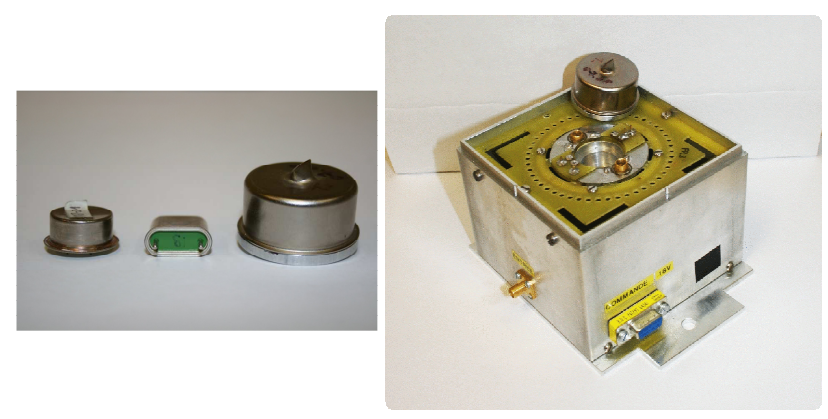

Figure 4. On left: various resonator cases (HC40, HC12, $5 \mathrm{MHz}$ BVA). On right: the lower part of one resoantor oven whose $\mathrm{HC} 40$ housing is visibly smaller than the $5 \mathrm{MHz}$ BVA enclosure lied aside.

\section{B. Task 2: Thermal effect}

It is well-known that temperature is a parameter of influence on the frequency stability, unavoidable in the design of an oscillator as well as in a passive measurement bench. Before beginning a study on a noise model based on a physical approach, temperature effects have to be evaluated at the $10^{-14}$ scale.

Two main causes of frequency changes due to temperature are usually identified:

- The first one is the well-known static effect linked to the real position of the operating point $T_{o p}$ versus the turn over point $T_{\text {top }}$ of the cubic frequency-temperature relationship.

The relative frequency sensitivity versus temperature fluctuations $\Delta \mathrm{T}$ around $T_{o p}$ can be written as:

$$
y=\frac{\Delta f}{f_{0}}=a_{T} \times \Delta T,
$$

with $a_{T}=\left[2 \beta+6 \gamma\left(T_{\text {top }}-T_{0}\right)\right] \cdot \delta T+3 \gamma \cdot \delta T^{2}$, where $\alpha, \beta$, $\gamma$ are respectively the first, second and third coefficients of the frequency-temperature relationship, depending on the cut angles, $T_{0}$ (usually $25^{\circ} \mathrm{C}$ ) is the reference temperature which 
corresponding to the reference frequency and $\delta T=T_{o p}-T_{t o p}$ is the temperature gap.

This is one of the most important adjustments to be done in an oven-controlled crystal oscillator (OCXO).

As an example, the usual SC cut whose controlledtemperature is set at $0.1 \mathrm{~K}$ (i.e. $\delta T=0.1 \mathrm{~K}$ ) from its turn-over point close to $T_{\text {top }}=85^{\circ} \mathrm{C}$, exhibits a coefficient value of $a_{T}=$ $10^{-9} \mathrm{~K}^{-1}$. This means that its temperature has to be controlled within $\Delta \mathrm{T}=100 \mu \mathrm{K}$ in order to get a frequency stability better than $10^{-13}$.

- The second cause of frequency changes linked to temperature has a dynamic origin and is commonly modeled by a phenomenological coefficient as:

$$
\frac{\Delta f}{f_{0}}=\tilde{a} \frac{d T}{d t}
$$

For the most simple SC cut resonator, the dynamic coefficient $\tilde{a}$ would be of a few $10^{-7} \mathrm{~K}^{-1} \mathrm{~s}$.

This phenomenon includes various effects such as:

- The thermal transfer function of the resonator, that is to say the ratio of the inner temperature of the vibrating volume, assumed to be homogeneous, and that of the crystal case also assumed to be homogeneous. This transfer function obviously depends on the mechanical mounting of the resonator according to whether the resonator is a BVA type or not for example, what are its thickness (depending on the selected overtone) and diameter, etc?

- Thermal gradients. Gradients into the resonator induce strains and then frequency shifts.

Anyway, all the possible origins of temperature changes are still to be identified at the $10^{-14}$ scale.

As previously mentioned, means used in our bench to filter and regulate the resonator temperature guarantee that ambient temperature changes are not a limiting factor of the resonator noise measurement. These means cannot be transposed directly in an oscillator structure because of their volume. So, the question of the resonator sensitivity to temperature at the $10^{-14}$ scale, in an oscillator, is still relevant. In addition, its series capacitor used for the frequency adjustment is also temperature-sensitive.

This is why; this study is the opportunity to review the theoretical aspect of temperature effects. It will be supported by a set of noise measurements on various types of resonators (conventional, QAS, BVA,...) for different remote-controlled operating temperature (the temperature step is $0.05^{\circ} \mathrm{C}$ ). This might permit to evaluate the limit of influence of temperature in oscillators, if it exists.

\section{Task 3: Influence of the tuning capacitor}

The resonator frequency has always to be tuned. Into each arm of the bench as in an oscillator, this is fulfilled by a series capacitor having a pull-up effect, here so-called tuning capacitor $C_{t}$ (see fig. 5): using the component definitions of fig. 5, the resulting frequency becomes with a good approximation,

$$
f_{\text {resonance }} \approx f_{s} \sqrt{1+\frac{C_{x}}{C_{0}+C_{t}}}
$$

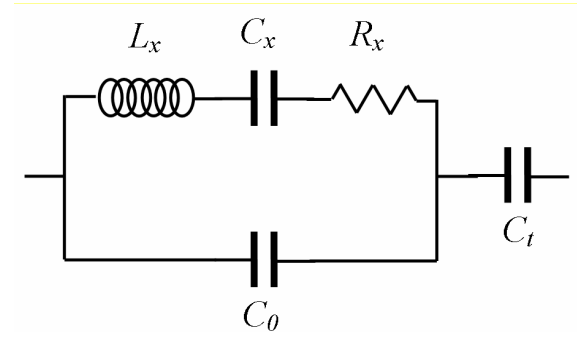

Figure 5. Electrical model of a crystal resonator whose motional parameters are $L_{x}, C_{x}$ and $R_{x} . C_{0}$ is its parallel capacitor whereas $C_{t}$ denotes the tuning series capacitor added to pull-up the overall resonant frequency.

In terms of relative frequency shift:

$$
\frac{\Delta f}{f_{s}} \approx \frac{C_{x}}{2\left(C_{0}+C_{t}\right)}
$$

where $f_{s}$ denotes the resonant frequency of the motional arm alone (i.e. $\left.L_{x} \cdot C_{x} \cdot\left(2 \pi f_{s}\right)^{2}=1\right)$, very close to the series frequency of the resonator. As an example, the addition of a tuning capacitor $C_{t}=100 \mathrm{pF}$ to a resonator exhibiting a parallel capacitor $C_{0}=2 \mathrm{pF}$, gives $\frac{\Delta f}{f_{s}} \approx 510^{-7}$.

Temperature sensitivity. This capacitor is obviously also temperature sensitive. Thus, it induces relative frequency changes around the resonant frequency (the pulled up frequency) such as:

$$
\frac{\partial f}{f_{\text {resonance }}} \approx k_{C t} \cdot \frac{d C_{t}}{C_{t}}
$$

As an illustration, numerical values of the coefficient $k_{C t}$ are shown in Fig. 6, in the case of a commercial $5 \mathrm{MHz}$ resonator. It takes values of a few $10^{-6}$. That means that a tuning capacitor which exhibiting a temperature coefficient of a few ppm / $\mathrm{K}$ will cause a relative frequency fluctuations versus temperature of a few $10^{-12} \mathrm{~K}^{-1}$.

In that case, a relative frequency stability of a few 10-14 could be reached provided that the tuning capacitor is temperature-controlled at better than $1 / 100 \mathrm{~K}$ around its operating temperature. This is not too much complicated to control but must be taken into account. 


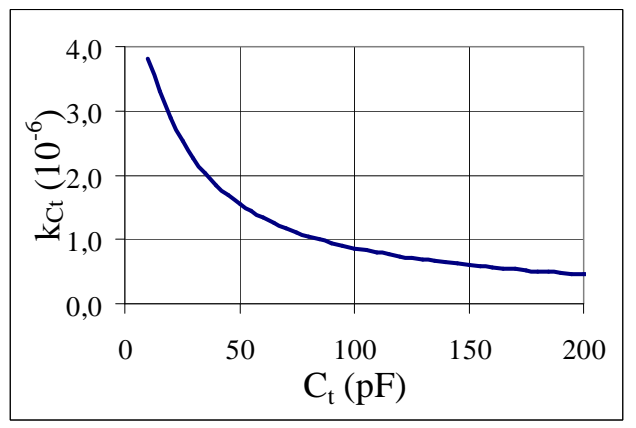

Figure 6. The coefficient $k_{C t}$ relating the relative frequency fluctuations and the relative tuning capacitor fluctuations, versus the capacitor value $C_{t}$.

Impedance sensitivity. The tuning capacitor modifies the overall impedance. In each arm of the bench, each resonator and its associated series capacitor are one element of a resistor bridge. Before beginning the noise measurement, one of the preliminary adjustments consists in tuning each series capacitor in order to get the resonance in both arms. When this operation is completed, the overall network of fig. 5 is equivalent to a resistance. In other words, its equivalent impedance $Z(f)=R(f)+X(f)$ is reduced to $Z\left(f=f_{\text {resonance }}\right)=R(f$ $\left.=f_{\text {resonance }}\right)=R_{\text {resonance }} \geq R_{x}$.

Nevertheless, $R_{\text {resonance }}$ is no more equal to the motional resistance $R_{x}$. Fig. 7 illustrates the influence of $\mathrm{C}_{\mathrm{t}}$ on $Z(f)$.

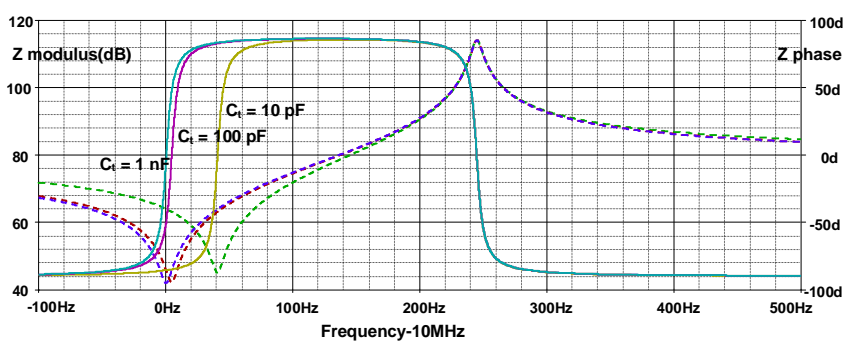

Figure 7. The modulus (in $\mathrm{dB}$ ) and the phase of the equivalent impedance $Z(f)$ of the network in fig. 5. At the resonance $Z=R_{\text {resonance }}$ (the minimum value of the modulus) depends on $C_{t}$.

This seems obvious but leads to some consequences on values and possible noise sources if fluctuations exist :

- The impedance matching is no longer true if resistors of the bridge have values previously set.

- The dissipated power into the resonator has to be evaluated again by taking into account some correction with regard to the simple resistor $R_{x}$.

- The real inner resonator temperature also depends on the real power dissipated into the resonator, as mentioned earlier.

- The loaded quality factor $Q_{L}$ is for $R_{\text {resonance }}>R_{x}$ and not $R_{x}$ (see fig. 8). Into the bench,

$$
Q_{L}=Q \cdot \frac{R_{\text {resonance }}}{R_{\text {resonance }}+R_{L}}
$$

where $Q$ is Q-factor of the system \{resonator + tuning capacitor\} (very close to the Q-factor of the resonator alone: see below), and $R_{L}$ is its global load resistor.

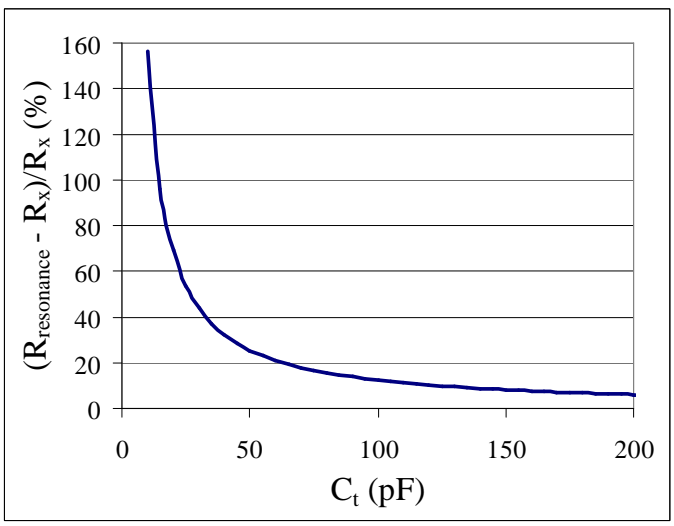

Figure 8. An example of the relative change of $\mathrm{R}_{\text {resonance }}$ versus $\mathrm{C}_{\mathrm{t}}$. One easily understands that the more the fluctuations of $\mathrm{C}_{t}$ the more the implied fluctuations of $\mathrm{R}_{\text {resonance. }}$

For the bench adjustments and calculations, one should be sure that these corrections are well applied. In addition, they could be noise sources if fluctuations exist. All of this is also applicable to oscillators.

Q-factor sensitivity. As previously mentioned, the loaded Qfactor depends on the real equivalent resistor $R_{\text {resonance }}$ of the system $\left\{\right.$ resonator $\left.+C_{t}\right\}$, which is a function of $C_{t}$, and on the quality factor $Q$ of this system. But, what about $Q$ versus $C_{t}$ ?

Fig. 9 shows an approximation of the relative phasefrequency slope $\frac{\dot{\varphi}-\dot{\varphi}_{x}}{\dot{\varphi}_{x}}$, where $\varphi=\operatorname{Arg} Z(f)$ close to $f_{\text {resonance }}$. Here the slope is denoted $\dot{\varphi}=\left.\frac{d \varphi}{d f}\right|_{\text {fresonance }}$ and $\dot{\varphi}_{x}=2 \frac{Q_{x}}{f_{s}}=2 \frac{L_{x} 2 \pi}{R_{x}}$ is the slope of the resonator without its tuning capacitor $C_{t}$.

One can observe that the value of $\frac{\dot{\varphi}-\dot{\varphi}_{x}}{\dot{\varphi}_{x}}$ never exceeds $110^{-3}$, for a conventional high quality resonator. This means that the Q-factor of the resonator plus its tuning capacitor $C_{t}$ remains close to the $\mathrm{Q}$-factor of the resonator alone, whatever the $C_{t}$ value.

Finally, in the frequency noise to phase noise conversion, the loaded Q-factor, $Q_{L}=Q \cdot \frac{R_{\text {resonance }}}{R_{\text {resonance }}+R_{L}}$, depends on 
$C_{t}$ only through $R_{\text {esonance, }}$ and not nearly through $Q$ which remains close to the resonator $\mathrm{Q}$-factor $Q_{x}$.

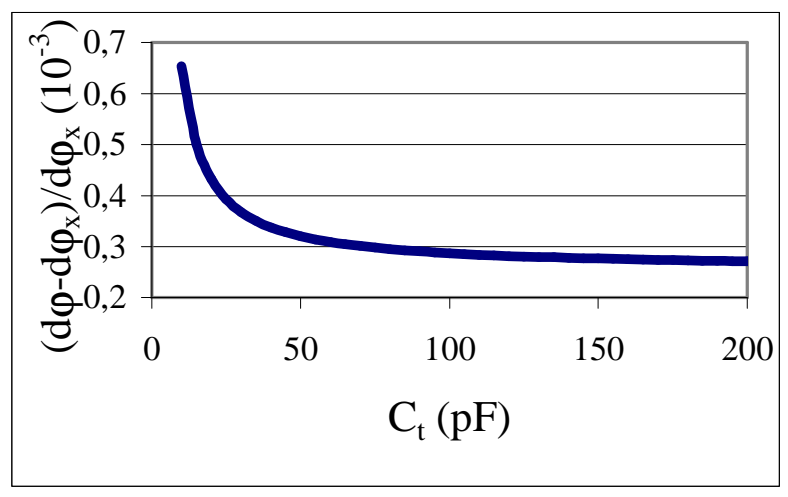

Figure 9. An example of the relative phase-frequency slope versus $C_{t}$, at the resonant frequency (which also depends on $C_{t}$ ).

\section{Task 4: load impedance sensitivity}

One can wonder about the influence of the mismatching of the bench with respect to the resonator impedance, in terms of resulting noise.

This topic is obviously closely related to that of the series capacitor sensitivity onto the resonator impedance as well as to that of the power sensitivity through the drive level dependency or more simply the non linear amplitudefrequency effect.

\section{E. Task 5: sensitivity of the resonator noise to the drive level.}

The injected power into both arms of the bench is adjustable. Thus this makes possible to excite the tested resonators in a broad power range covering the operating range of those resonators in oscillators (typically a few tens of microwatts).

The correlation between noise and drive level, if it exists, could be established in such a way, for various types of resonators.

Nevertheless, one can notice that changing the power dissipated into the resonator induces changes of its inner temperature and changes of the temperature gradients, that is to say, as a consequence its resonant frequency (see $\S$ "B. Task 2: Thermal effect").
For high drive levels, a non linear effect, the so-called amplitude-frequency effect, also interacts on the frequency value.

When changing the power level, many possible causes of frequency drift and frequency disturbances have then to be considered (if possible!).

On very low drive level (typically $-100 \mathrm{dBm}$ ), measurements of motional parameters will also allow continuing the investigations concerning the correlation between the drive level dependency (DLS) and noise of resonators [5]

\section{CONCLUSION}

Since a few years, quartz crystal oscillators seem to have reached their limit in terms of frequency stability. They still are attractive for their volume, relatively low consumption in steady state, especially for space applications.

The goal of this program is to determine and understand the mechanisms responsible of current limitation of resonator noise in order to reduce their susceptibility and to evaluate their potentiality in a short and mean future.

To reach this goal, this program federates several major French and European entities.

\section{REFERENCES}

[1] E. Rubiola, V. Giordano, "On the 1/f frequency noise in ultra-stable quartz oscillators," IEEE Transact. Ultrason. Ferroelec. Freq. Contr. vol. 54 no. 1 pp. 15-22, January 2007. Preprint available on http://rubiola.org and http://arxiv.org, document arXiv:physics/0602110.

[2] F. Sthal, X. Vacheret, S. Galliou, P. Salzenstein, E. Rubiola, G. Cibiel, "Advanced bridge instrument for the measurement of the phase noise and of the short-term frequency stability of ultra-stable quartz resonators", in these proceedings.

[3] R. J. Besson, M. Mourey, S. Galliou, F. Marionnet (ENSMM France), F. Gonzalez, P. Guillemot (CNES France), R. Tjoelker, W. Diener, A. Kirk (JPL USA), "10 MHz Hyperstable Quartz Oscillators Performances", joint meeting of the 13th European Fequency and Time Forum and 1999 IEEE International Frequency Control Symposium, Besançon France, pp 326-330, vol.1, May 1999.

[4] F. Sthal, S. Galliou, P. Abbé, N. Franquet, X. Vacheret P. Salzenstein, E. Rubiola and G. Cibiel, " Thermal characterization of crystal ovens used in phase noise measurement system ", Proc. IEEE Int. Freq. Cont. Symp., Miamai, Florida, 5-7 June, pp 736-739, 2006.

[5] R. Brendel, M. Addouche, R. Brendel, E. Rubiola, G. Cibiel "Low drive level sensitivity (DLS) of quartz crystal resonators. " Proceedings of the 20th European Frequency and Time Forum, Braunschweig, Germany, pp.105-112, March 2006 